\title{
Associations of Individual and Joint Expressions of ERCC6 and ERCC8 with Clinicopathological Parameters and Prognosis of Gastric Cancer
}

\section{Jing Chen}

Tumor Etiology and Screening Department of Cancer Institute and General Surgery, the First Hospital of China Medical University, Shenyang 110001, China. Key Laboratory of Cancer Etiology and Prevention in Liaoning Education Department, the First Hospital o

\section{Liang Li}

Tumor Etiology and Screening Department of Cancer Institute and General Surgery, the First Hospital of China Medical University, Shenyang 110001, China. Key Laboratory of Cancer Etiology and Prevention in Liaoning Education Department, the First Hospital o

\section{Li-Ping Sun}

Tumor Etiology and Screening Department of Cancer Institute and General Surgery, the First Hospital of China Medical University, Shenyang 110001, China. Key Laboratory of Cancer Etiology and Prevention in Liaoning Education Department, the First Hospital o

\section{Yuan Yuan}

Tumor Etiology and Screening Department of Cancer Institute and General Surgery, the First Hospital of China Medical University, Shenyang 110001, China. Key Laboratory of Cancer Etiology and Prevention in Liaoning Education Department, the First Hospital o

\section{Jing-jing Jing ( $\sim$ hellojjjing@163.com )}

The First Affiliated Hospital of China Medical University https://orcid.org/0000-0002-9807-8089

\section{Research Article}

Keywords: gastric cancer, prognosis, clinicopathological parameters, ERCC6, ERCC8, expression

Posted Date: February 4th, 2021

DOI: https://doi.org/10.21203/rs.3.rs-117489/v2

License: @ (1) This work is licensed under a Creative Commons Attribution 4.0 International License. Read Full License 


\section{Abstract}

\section{Background}

Excision repair cross-complementing group 6 and 8 (ERCC6 and ERCC8) have been implicated in ailments such as genetic disease and cancer. However, the relationship between individual and joint expressions of ERCC6/ERCC8 and clinicopathological parameters as well as prognosis of gastric cancer (GC) still remains unclear.

\section{Methods}

In this study, protein expressions of ERCC6, ERCC8 and ERCC6-ERCC8 were detected by immunohistochemistry (IHC) with 109 paired GC and para-cancerous normal tissue samples. IHC results and RNA-seq data extracted from The Cancer Genome Atlas (TCGA) were used to explore the clinical value of ERCC6 and ERCC8 expression in GC. We further conducted protein-protein interaction analysis, Gene Ontology, Kyoto Encyclopedia of Genes and Genomes, gene set enrichment analysis, and gene-gene interaction analysis for the exploration of the function and regulation network of ERCC6 and ERCC8 in GC.

\section{Results}

Individual and joint ERCC6/ERCC8 expression were significantly higher in adjacent normal mucosa compared with GC tissues. Protein expressed levels of ERCC6, ERCC8, double negative ERCC6-ERCC8 and double positive ERCC6-ERCC8 and overexpressed ERCC6 mRNA were related to better clinicopathologic parameters, while overexpressed ERCC8 mRNA suggested worse parameters. Univariate survival analysis indicated an increased OS with higher ERCC6 protein expression and ERCC8 mRNA expression, and a decreased OS with double negative ERCC6-ERCC8 expression. Bioinformatic analyses showed ERCC6 and ERCC8 were associated with nucleotide excision repair (NER) pathway, and six and ten gene sets were figured out to be related with ERCC6 and ERCC8, respectively. Direct physical interactions were found between ERCC6 and ERCC8.

\section{Conclusions}

Individual and joint expressions of ERCC6/ERCC8 were associated with clinical features of GC. Expressed levels of ERCC6 and double negative ERCC6-ERCC8 protein, and ERCC8 mRNA were related to prognosis of GC. ERCC6 and ERCC8 primarily function in the NER pathway, and could regulate GC progression through the regulation of $\mathrm{PI} 3 \mathrm{~K} / \mathrm{AKT} / \mathrm{mTOR}$ pathway.

\section{Background}

The human genome is under a condition where DNA impairment and correction are in a dynamic equilibrium relationship. A variety of DNA repair mechanisms have been found to help maintain the integrity of genes damaged by endogenous and exogenous variables(1). Nucleotide excision repair (NER) can repair a wide range of DNA lesions, including oxidatively damaged DNA bases, bulky adducts and UV-induced cyclobutane pyrimidine dimers $(2,3)$. The repair pathway cannot be activated with deficient expression of DNA repair genes, which can lead to a decrease in DNA repair capacity and an increase in cancer susceptibility(4). 
Excision repair cross-complementing group 6 (ERCC6/CSB) and excision repair cross-complementing group 8 (ERCC8/CSA) are core members vital for NER pathway(5-8). Considering their important function in the NER pathway, many scholars conducted experiments to explore the roles of ERCC6 and ERCC8 in disease onset and progression. Results of these experiments showed that expression of ERCC6 and ERCC8 had clinical significance in ailments such as genetic disease and cancer. Cheng et al. $(9,10)$ observed in their studies that patients with lung and head and neck cancer were more likely to have decreased ERCC6 mRNA expression in comparison with controls. In sharp contrast, overexpressed ERCC6 mRNA was detected in colorectal cancer compared with matched normal tissues(11), and Caputo et al.(12) have convincingly reported higher ERCC6 mRNA expression in kidney and lung cancer samples, and western blot analysis revealed increased ERCC6 protein levels in bladder, cervix, prostate and breast cancer cells compared with normal cells. Previous researches have also revealed that ERCC6 and ERCC8 play a crucial part in the onset and progression of a hereditary disease called Cockayne syndrome(13). It has been observed in our previous study that ERCC6, ERCC8, and ERCC6-ERCC8 joint expression is related to the risk of gastric cancer (GC)(14).

All current data turn out to indicate the fact that there exist heterogeneous expression of ERCC6 and ERCC8 in disease, and thus influencing the development and progression of disease. However, to date no prior reports have recorded the prognostic implications of ERCC6, ERCC8 and ERCC6-ERCC8 expressions in GC. For the first time, our study conducted a comprehensive analysis using immunohistochemistry (IHC) and RNA-seq data to explore the associations between ERCC6 and ERCC8 expression and clinicopathological parameters and prognosis of GC.

\section{Materials And Methods}

\section{Human tissue specimens}

Paired gastric cancer and para-cancerous normal tissue samples were collected from 109 individuals with gastric cancer, who were diagnosed at the anorectal department of the First Hospital of China Medical University. Each selected patient had not received neoadjuvant chemoradiotherapy or any other treatment from 2012 to 2015 . Histological diagnoses were completed following the updated Sydney System

Classification(15) and the World Health Organization criteria(16) for gastritis and GC, respectively. The 2010 7th edition of the TNM staging system of the International Union Against Cancer/American Joint Committee on Cancer was selected to stage the tumor(17), based on postoperative pathologic examination. This study was approved by the ethics committee of the First Hospital of China Medical University. Written informed consent was obtained from all participants.

\section{Immunohistochemistry staining and evaluation}

Every single paraffin-embedded sample was sectioned into a 4- $\mu \mathrm{m}$-thick slide. All the deparaffinized and rehydrated slides were boiled at $95^{\circ} \mathrm{C}$ for 30 minutes in citrate antigen retrieval solution $(\mathrm{pH} 6.0)$. Immunohistochemistry (IHC) staining was performed with the avidin-biotin complex method as our previous study described(14). CSB (ERCC6) rabbit polyclonal antibody (1:300 dilution, Origene, TA313375, USA) and AB1(anti-ERCC8) antibody produced in rabbit (1:500 dilution, Sigma, AV31542, USA) were used. 
Two experienced pathologists who were blinded to patient-related information evaluated and scored the staining results independently. A semi-quantitative scoring criterion was applied to analyze the staining extent of each slide(18). The staining score was categorized on the basis of intensity: 0 - no staining, 1 - light staining, 2 - moderate staining and 3 - strong staining and coloring ratio: 0 ( $\leq 5 \%), 1(5 \%-25 \%), 2(25 \%-50 \%), 3(50 \%-$ $75 \%), 4$ ( $\geq 75 \%$ ) of the IHC results. An immunoreactivity score (IS) was generated by multiplying the two scores for each sample. All the scores were applied to indicate certain extent of positive staining except the score of 0 , which suggested a negative protein expressed level.

\section{Clinical information and RNA-seq data collection}

Data of $109 \mathrm{IHC}$ cases concerning age, gender, smoking, family history and alcohol consumption were obtained via questionnaire. Clinical characteristics were collected from medical records, including TNM stage, Lauren's classification, Borrmann classification, tumor size, phase of progression, lymph node metastasis, perineural invasion, and vascular invasion. The final follow-up was completed on July 2016. Full data concerning prognosis were obtained from 97 participants.

Data of 415 stomach cancer patients, which included RNA-seq data and survival information, were downloaded from TCGA (https://cancergenome.nih.gov/)(19, 20). Detailed information of clinicopathological parameters including age, gender, grade, TNM stage and histological type were also downloaded for further analyzing.

\section{Interaction and functional analysis of ERCC6 and ERCC8}

Given the clinical significance of ERCC6/ERCC8 in GC, we then investigated the biological functions of ERCC6 and ERCC8. First we used ERCC6 and ERCC8 as core genes to construct protein-protein interaction (PPI) networks by Search Tool for the Retrieval of Interacting Genes (STRING v.11.0; https://string-db.org/; accessed on August 27, 2020), to mine proteins that have functional interactions with ERCC6/ERCC8(21). Analytic information including nodes degrees and biological networks was visualized with Cytoscape platform (v.3.7.2) (22). And ten most associated proteins were showed in the diagrams.

Then we conducted enrichment analyses of Gene ontology (GO) and Kyoto Encyclopedia of Genes and Genomes (KEGG) to explore the biological functions of ERCC6 and ERCC8 with the Database for Annotation, Visualization and Integrated Discovery (DAVID; v.6.8; https://david.ncifcrf.gov/home.jsp; accessed on August $31,2020)$, a user friendly database providing comprehensive analysis of gene annotation(23). $R$ language (Version 3.6.3) and the ggplot2 package were applied to visualize the analytic results. Terms with a $P<0.05$ were deemed significant and for $\mathrm{GO}$, only top ten terms of each group were selected to be visualized.

\section{Identification of regulation networks of ERCC6 and ERCC8 by GSEA}

Gene set enrichment analysis was conducted on the GSEA platform (version 4.1.0; https://www.broadinstitute.org/gsea/) coupled with MSigDB database $(24,25)$. Oncogenic signature gene sets (c6.all.v7.1.symbols.gmt) and obtained TCGA expression data were included in this analysis. Through GSEA, we could clarify the regulation networks of which ERCC6 and ERCC8 were involved in GC. Significantly changed regulation networks were identified through a thousand times of phenotype permutation test and the metric for the analysis was set as pearson. A normalized $p$ value $<0.01$ and a false discovery rate (FDR) less than 0.25 
were selected as criteria for significant enrichment results. Normalized enriched score (NER) was applied to rank the obtained results.

\section{Identifying gene-gene interactions between ERCC6 and ERCC8}

To identify interactions between ERCC6 and ERCC8, we performed gene-gene interaction analysis with the Gene Multiple Association Network Integration Algorithm (GeneMANIA; https://www.genemania.org/; accessed on November 13,2020), which is a user-friendly interface providing analysis with available genomics and proteomics data(26). To display interactions, nodes and links represented genes and networks respectively in the visualized results.

\section{Statistical analysis}

IBM SPSS Statistics for Windows, version 23.0 (IBM Corp., Armonk, N.Y., USA) and R platform (Version 3.6.3) were used for statistical analyses. For IHC, Pearson $\chi^{2}$ test was used when analyzing the correlations between ERCC6 and ERCC8 expressed levels and clinicopathological parameters; univariate and multivariate Cox regression analyses have been selected for the determination of their impact on overall survival (OS), and variables including age, TNM stage, perineural invasion, vascular invasion and lymph node metastasis were further adjusted in the multivariate model to evaluate the independent prognostic value. For RNA-seq data, Wilcoxon test and Kruskal-Wallis $\mathrm{H}$ test have been employed when calculating the interrelationships between ERCC6/8 expressions and clinical characteristics; two-sided Log-rank test and multivariate Cox proportionalhazards model adjusted by gender, age, grade, stage, T, N, and $\mathrm{M}$ were selected to clarify the prognostic value of ERCC6 and ERCC8. A $P<0.05$ suggested statistical difference.

\section{Results}

\section{Expression of ERCC6 and ERCC8 in GC and adjacent normal mucosa}

In this study we compared the expressed levels of ERCC6 and ERCC8 between GC and adjacent normal mucosa. Representative ERCC6 and ERCC8 staining were present in Figure 1. Our results suggested that individual and joint expressions of ERCC6 and ERCC8 were obviously higher in adjacent normal mucosa than in GC tissues (all $P<0.001$ ) (Table 1). Specifically, the ERCC6-ERCC8 double positive rate dropped to $16.5 \%$ in GC and the double negative rate was only $1.8 \%$ in adjacent normal mucosa. 
Table 1. Protein expression of ERCC6/ERCC8 in different gastric tissues

\begin{tabular}{|c|c|c|c|c|c|c|c|c|}
\hline & \multicolumn{8}{|c|}{ Adjacent SG vs GC } \\
\hline & \multicolumn{2}{|c|}{ ERCC6 } & \multicolumn{2}{|c|}{ ERCC8 } & \multicolumn{2}{|c|}{ ERCC6-ERCC8* } & \multicolumn{2}{|c|}{ ERCC6-ERCC8** } \\
\hline $\begin{array}{c}\text { Positive } \\
\text { (\%) }\end{array}$ & $105(96.3)$ & $44(40.4)$ & $104(95.4)$ & $42(38.5)$ & 102(93.6) & $18(16.5)$ & $107(98.2)$ & $60(55.0)$ \\
\hline $\begin{array}{l}\text { Negative } \\
\text { (\%) }\end{array}$ & $4(3.7)$ & $65(59.6)$ & $5(4.6)$ & $67(61.5)$ & $7(6.4)$ & $91(83.5)$ & $2(1.8)$ & $49(45.0)$ \\
\hline $\mathbf{P}$ & \multicolumn{2}{|c|}{$<0.001$} & \multicolumn{2}{|c|}{$<0.001$} & \multicolumn{2}{|c|}{$<0.001$} & \multicolumn{2}{|c|}{$<0.001$} \\
\hline
\end{tabular}

GC, gastric cancer; SG superficial gastritis; * double positive for ERCC6 and ERCC8 expression; ** double negative for ERCC6 and ERCC8 expression Correlations of ERCC6 and ERCC8 expression with clinicopathological characteristics in GC

We explored the associations of ERCC6/ERCC8 expressed levels with clinicopathological parameters in GC patients and the results were summarized in Supplementary file 1: table S1. ERCC6 expression was significantly related to Borrmman classification $(P=0.017)$, Lauren's classification $(P=0.004)$, TNM stage $(P=0.005)(P=0.012$ for T stage) and perineural invasion $(P=0.001)$. High ERCC6 expression was observed in gastric cancers of Borrmman class I-II, TNM stage I-II, intestinal-type and without perineural invasion. Expression of ERCC8 was statistically higher in study cohort with TNM stage I-II in comparison to stage III-IV $(P<0.001)(P<0.001$ for T stage; $P=0.002$ for lymph node metastasis), and was higher in cancer with earlystage and small size ( $P=0.031$ and 0.007 , respectively). Higher expression of ERCC8 was also observed in intestinal type GC (P=0.008). As for the joint expression of ERCC6/ ERCC8, double positivity was related to small tumor size $(P=0.005)$, Borrmman I-II stage $(P<0.001)$, TNM I-II stage $(P=0.001)(P<0.001$ for T stage), Lauren intestinal type $(P=0.014)$ of $G C$ and negative perineural invasion $(P=0.015)$. Double negativity was associated with TNM III-IV stage $(P<0.001)(P=0.002$ for T stage), positive perineural invasion $(P=0.002)$, advanced stage $(P=0.034)$ and diffuse type $(P<0.001)$ of $G C$.

Analysis results of RNA-seg data obtained from TCGA was shown in Figure 2, higher ERCC6 expression was related with better T stage $(P=0.027)$, which is consistent with the $\mathrm{IHC}$ analysis results, while no statistical results were found between ERCC6 expression and age $(P=0.570)$, gender $(P=0.646)$, histological type $(P=$ $0.425)$, grade $(P=0.072)$, stage $(P=0.091), N$ stage $(P=0.572)$ and $M$ stage $(P=0.242)$. As for ERCC8, Figure 3 revealed that overexpressed ERCC8 was closely related to worse grade $(P=0.018)$, advanced stage $(P=0.022)$, and worse $N$ stage $(P=0.037)$; the associations between ERCC8 expression and age, gender, histological type, T stage and M stage were not significant ( all $\mathrm{P}>0.05$ ).

\section{The relationship between ERCC6 and ERCC8 expression and prognosis of GC}

As shown in table 2, univariate survival analysis showed no significant correlation between protein expressed levels of ERCC8 and GC prognosis $(P=0.211)$, while a significant correlation was observed between ERCC6 protein expressed levels and GC prognosis( $P=0.047, \mathrm{HR}=3.416,95 \% \mathrm{Cl}=1.017-11.475)$. In addition, double negative expression of ERCC6 and ERCC8 was significantly associated with poorer prognosis $(\mathrm{P}=0.022, \mathrm{HR}=2.603,95 \% \mathrm{Cl}=1.148-5.905)$. However, no statistical association was found between the expressed levels of ERCC8 and ERCC6-ERCC8 and GC prognosis (both $P>0.05)$. Because TNM stage $(P<0.001)$, age $(P=0.039)$, perineural invasion $(P=0.043)$, vascular invasion $(P=0.007)$, and lymph node metastasis $(P<0.001)$ are all statistically associated with gastric cancer prognosis (Supplementary file 2: table S2), Cox's proportional hazards model adjusted by perineural invasion, TNM stage, age and vascular invasion was further applied to evaluate the prognostic value. However, the multivariate analysis suggested that ERCC6 or ERCC8 or ERCC6-ERCC8 expressed level was not an independent factor for GC prognosis (all $\mathrm{P}>0.05$ ) (Table 2).

Survival analysis with RNA-seq data suggested that higher ERCC8 mRNA expression was related to better OS $(P=0.025$; Fig.3I) while ERCC6 expression made no sense $(P=0.15$; Fig. $2 \mathrm{I})$. And multivariate Cox proportional hazard models showed no significant results with ERCC6 $(\mathrm{P}=0.969 ; \mathrm{HR}=0.995 ; 95 \% \mathrm{Cl}=$ $0.761-1.300)$ and ERCC8 ( $\mathrm{P}=0.078 ; \mathrm{HR}=1.393 ; 95 \% \mathrm{Cl}=0.964-2.014)$.

\section{Function analysis for ERCC6 and ERCC8}

To explore the function of ERCC6 and ERCC8, first we constructed PPI networks using ERCC6 and ERCC8 as core genes. With a confidence score of more than 0.4, Figure 4A showed that ten most associated proteins of ERCC6 were ERCC2, ERCC3, ERCC4, ERCC5, ERCC8, TP53, XPC, XPA, POLR2A and POLR2I; and for ERCC8, the proteins were XAB2, DDB1, ERCC3, ERCC4, ERCC2, CUL4A, UVSSA, ERCC5, XPA and GTF2H2 (Figure 4B). 
Then we conducted analyses of GO and KEGG according to the network results we obtained from String. As revealed in Figure 5, ERCC6 network genes showed enrichment in molecular functions of protein N-terminus binding, damaged DNA binding and DNA-dependent ATPase activity (Figure 5A). They were mainly involved in nucleoplasm, transcription factor TFIID complex, and holo TFIIH complex according to cellular components analysis result (Figure 5B). Figure 5C showed that ERCC6-interactive genes were significantly enriched in biological processes of nucleotide-excision repair and UV protection. Analysis results of KEGG suggested that these genes were closely related to nucleotide excision repair, Huntington's disease, RNA polymerase and basal transcription factors (Figure 5D). As for ERCC8 network genes, results of GO enrichment analysis showed that these genes were related to the composition of transcriptional initiation complexes and ubiquitin ligase complexes, and not surprisingly, their main molecular functions and participated biological processes bore a remarkable resemblance to ERCC6 network genes (Figure 6A-C). Similar KEGG results, nucleotide excision repair and basal transcription factors were also observed in ERCC8 network genes. And a special part of this analysis results was ubiquitin mediated proteolysis (Figure 6D).

\section{Identification of gene sets associated regulatory networks of ERCC6 and ERCC8}

Further we identified most positively and negatively related gene sets with ERCC6 and ERCC8, to figure out the cellular regulatory networks in GC that ERCC6 and ERCC8 were involved in. Oncogenic signatures analysis indicated that in GC there existed six and ten most significant gene sets for ERCC6 and ERCC8, respectively. Among the results, both ERCC6 and ERCC8 were associated with TBK1 and BCAT associated cellular regulatory networks; ERCC6 was also associated with regulated by EIF4, MTOR, JAK2 and CSR related regulatory networks; ERCC8 was also associated with PIGF, RB, ERBB2, GCNP, SRC and CYCLIN D1 related regulatory networks. Detailed information are shown in Figure 7 for ERCC6 and Figure 8 for ERCC8.

\section{Gene-gene interaction network between ERCC6 and ERCC8}

Gene-gene interaction network accessed from GeneMANIA clarified the correlations of ERCC6 and ERCC8 among pathway, predicted, shared protein domains, physical interactions, co-localization, and co-expression. As shown in Figure 9, there existed direct interactions including physical interactions and pathway and indirect interactions including prediction, co-expression, colocalization and shared protein domains between ERCC6 and ERCC8.

\section{Discussion}

By analyzing IHC and TCGA data, our experiment elucidated that abnormally expressed ERCC6 and ERCC8 were associated with clinicopathological behaviors and survival of GC. Furthermore, by performing bioinformatics analysis of GO, KEGG, GSEA and gene-gene interaction analysis, our research extended the existing knowledge of ERCC6/ERCC8 in GC.

We initially detected protein expressed levels of ERCC6/ERCC8 in GC and para-cancerous tissues. The results indicated that their expressions were significantly decreased in GC, in comparison to adjacent tissues despite individual or joint expression. Then we investigated associations between protein expression of ERCC6 and ERCC8 and clinicopathological parameters, and the analysis showed that overexpressed ERCC6, ERCC8 and ERCC6-ERCC8 were significantly related to favorable clinicopathological features, which are key factors that have great impact on disease progression. RNA-seq data revealed identical results with ERCC6 that higher ERCC6 expression was associated with favorable T stage, while overexpressed ERCC8 was associated with unfavorable clinicopathological parameters. We suspected that the discrepancy may be due to some potential mechanisms that resulted in the instability of ERCC8 protein in GC progression. And cancer cells lacking ERCC6 or ERCC8 protein, which are responsible for DNA repair, may exhibit a more malignant and poorly differentiated phenotype. A recent research reported that ERCC6 deficiency could result in heterochromatin loss and exacerbates cellular aging (27). Defects in ERCC6 and ERCC8 will influence the coupling of transcription and repair to a certain extent, thus leading to declining DNA repair capacity(28). Physiologically, DNA repair capacity could be related to expression levels of proteins involved in DNA repair activities(29). Previous studies have also reported that a downregulation of DNA repair genes is related to late stage cancers and malignant transformation(30). Therefore, it is conceivable that the expressed status of DNA repair genes could reflect the capacity of a cell to meet repair demands after being stimulated by a carcinogen. We suggested that ERCC6 and ERCC8 downregulation could induce persistent existence of unrepaired DNA lesions, leading to decreased DNA repair capacity and increased cancer susceptibility, and finally resulting in cancer progression.

Further to explore the prognostic value of ERCC6 and ERCC8, we studied the correlation between ERCC6 and ERCC8 expression and survival in GC patients with both IHC and RNA-seq data. According to univariate survival analysis based on IHC, higher ERCC6 protein expression was associated with better prognosis while

Page $7 / 20$ 
double negative ERCC6 and ERCC8 expression indicated worse overall survival of GC patients. And RNA-seq data also showed that overexpressed ERCC8 was related to a better OS of GC patients. When adjusting for certain parameters in the Cox multivariate analysis, analyses results of ERCC6 and ERCC8 expression with IHC and RNA-seq data no longer maintained independent predictive power, which may be due to the complexity of tumor progression. A previous lab study showed that knockdown of ERCC6 could sensitize HCT116 cells to 5-Fluorouracil in xenograft mouse models and colorectal cancer patients with high ERCC6 expression exhibited shorter overall survival(31). As for other DNA repair family genes, high ERCC5 expression was shown to correlate with shorter survival times compared with low ERCC5 expression(32), whereas decreased ERCC1 expression was reported to predict a favorable prognosis in gastric cancer(33). Overall, our data suggested that expressed levels of ERCC6 and double negative ERCC6-ERCC8 protein, and ERCC8 mRNA, to some extent, may possess potential prognostic value in GC, and some certain factors should also be taken into account to estimate GC prognosis more comprehensively in the further analysis.

Next, bioinformatic analyses were conducted to better investigate biological functions and regulation networks of ERCC6 and ERCC8 in GC progression. First we queried the 10 most relevant genes of ERCC6 and ERCC8 through String and then performed GO and KEGG analyses with the obtained results. Enrichment analysis of ERCC6 and ERCC8 and their relevant genes showed similar results. Both the two genes were mainly involved in the composition of transcriptional initiation complexes and exerted influences on diverse nucleotide excision repair pathways. Similarly in other experiments, researchers have identified ERCC6 and ERCC8 as core NER genes(34-36). KEGG pathway analysis results further revealed that ERCC6 also functioned in Huntington's disease and ERCC8 showed significant impacts in ubiquitin mediated proteolysis. Consistent with our analyses, one study have reported that ERCC8 are involved in the formation a complex which exhibits ubiquitin ligase activity(37). Furthermore, we conducted GSEA analysis to explore ERCC6 and ERCC8 associated regulation networks in GC. Usually a set of genes which exhibit certain patterns of up or downregulation when an already known pathway related to tumorigenesis is activated, are defined as an oncogenic pathway signature. Here in our study, oncogenic signatures analysis suggested that ERCC6 was significantly associated with the oncogenic signatures of EIF4E, TBK1, BCAT, MTOR, JAK2 and CSR related regulation networks. As for ERCC8, the results indicated a significant relationship with TBK1, PIGF, BCAT, RB, ERBB2, GCNP, SRC and CYCLIN_D1 associated oncogenic regulation networks. These days emerging evidence has illustrated that $\mathrm{PI} 3 \mathrm{~K} / \mathrm{AKT} / \mathrm{mTOR}$ pathway deregulation plays an important part in GC progression(38). Currently, one study conducted by Riquelme mentioned that two mTOR pathway genes, EIF4E and MTOR, were overexpressed in GC cells(39). It has been found that ERBB2 could mediate the activation of PI3K(40). Moreover, some studies have reported the environment-dependent inhibition or activation role of TBK1 in mTOR signaling(41-43). Therefore, given all the above results, we suspected that ERCC6 and ERCC8 could regulate GC progression through the regulation of PI3K/AKT/mTOR pathway. Because of the similar and identical functions and pathways found in our analysis, we then did gene-gene interaction analysis to figure out the potential associations between ERCC6 and ERCC8. The results demonstrated that there did exist direct physical interactions and pathways between ERCC6 and ERCC8, which was supported by one previous study(44). And indirect interactions including prediction, coexpression, colocalization and shared protein domains were also revealed in the results. From these we suggested the existence of alliance mechanisms between ERCC6 and ERCC8, which needs further in-depth study.

\section{Conclusions}

In conclusion, individual and joint expressions of ERCC6 and ERCC8 were associated with clinical features of GC. Protein expressed levels of ERCC6 and double negative ERCC6-ERCC8, and ERCC8 mRNA expression were related to prognosis of GC patients. ERCC6 and ERCC8 primarily function in the NER pathway, and could regulate GC progression through the regulation of PI3K/AKT/mTOR pathway. Direct physical interactions existed between ERCC6 and ERCC8. However, a larger cohort is in need for the validation of these conclusions, and the mechanisms underlying these results warrant further experimental investigations.

\section{Abbreviations}

ERCC6/CSB: excision repair cross-complementing group 6; ERCC8/CSA: excision repair crosscomplementing group 8; GC: gastric cancer; IHC: immunohistochemistry; TCGA: The Cancer Genome Atlas; NER: nucleotide excision repair; PPI: protein-protein interaction; STRING: Search Tool for the Retrieval of Interacting Genes; GO: Gene ontology; KEGG: Kyoto Encyclopedia of Genes and Genomes; DAVID: Database for Annotation, Visualization and Integrated Discovery; GSEA: Gene set enrichment analysis; FDR: false discovery rate; NER: normalized enriched score; OS: overall survival. 


\section{Declarations}

\section{Ethics approval and consent to participate}

This study was approved by the Ethics Committee of the First Affiliated Hospital of China Medical University Shenyang, China. Informed consent was considered to be exempt after review by the Ethics Committee.

\section{Consent for publication}

Not applicable.

\section{Availability of data and materials}

The data that support the results of this study are available within the article.

\section{Competing interests}

The authors declare that they do not have a commercial or other association that might pose a conflict of interest.

\section{Funding}

The authors gratefully acknowledge financial grants from the Key Project of the Natural Science Foundation of Liaoning Province (Ref No. 20180540037).

\section{Authors' contributions}

YY contributed to study design and made significant revisions on the drafts. JJ-J, LL and JC completed the experiments. JJ-J and JC performed the statistical analyses. JJ-J, LS and JC drafted the paper. JC conducted the bioinformatics analyses. All authors read and approved the final manuscript.

\section{Acknowledgements}

Not applicable.

\section{References}

1. Lindahl T, Wood RD. Quality control by DNA repair. Science (New York, NY). 1999;286(5446):1897-905.

2. Marteijn JA, Lans $\mathrm{H}$, Vermeulen W, Hoeijmakers JH. Understanding nucleotide excision repair and its roles in cancer and ageing. Nature reviews Molecular cell biology. 2014;15(7):465-81.

3. Cleaver JE, Lam ET, Revet I. Disorders of nucleotide excision repair: the genetic and molecular basis of heterogeneity. Nat Rev Genet. 2009;10(11):756-68.

4. O'Driscoll M. Diseases associated with defective responses to DNA damage. Cold Spring Harb Perspect Biol. 2012;4(12).

5. Hanawalt PC, Spivak G. Transcription-coupled DNA repair: two decades of progress and surprises. Nature reviews Molecular cell biology. 2008;9(12):958-70.

6. Enoiu M, Jiricny J, Schärer OD. Repair of cisplatin-induced DNA interstrand crosslinks by a replicationindependent pathway involving transcription-coupled repair and translesion synthesis. Nucleic acids research. 2012;40(18):8953-64.

7. Iyama T, Lee SY, Berquist BR, Gileadi O, Bohr VA, Seidman MM, et al. CSB interacts with SNM1A and promotes DNA interstrand crosslink processing. Nucleic acids research. 2015;43(1):247-58.

8. Vermeulen W, Fousteri M. Mammalian transcription-coupled excision repair. Cold Spring Harb Perspect Biol. 2013;5(8):a012625.

9. Cheng L, Spitz MR, Hong WK, Wei Q. Reduced expression levels of nucleotide excision repair genes in lung cancer: a case-control analysis. Carcinogenesis. 2000;21(8):1527-30.

10. Cheng L, Sturgis EM, Eicher SA, Spitz MR, Wei Q. Expression of nucleotide excision repair genes and the risk for squamous cell carcinoma of the head and neck. Cancer. 2002;94(2):393-7.

11. Yu J, Mallon MA, Zhang W, Freimuth RR, Marsh S, Watson MA, et al. DNA repair pathway profiling and microsatellite instability in colorectal cancer. Clinical cancer research : an official journal of the American Association for Cancer Research. 2006;12(17):5104-11. 
12. Caputo M, Frontini M, Velez-Cruz R, Nicolai S, Prantera G, Proietti-De-Santis L. The CSB repair factor is overexpressed in cancer cells, increases apoptotic resistance, and promotes tumor growth. DNA repair. 2013;12(4):293-9.

13. Friedberg EC. How nucleotide excision repair protects against cancer. Nat Rev Cancer. 2001;1(1):22-33.

14. Jing JJ, Lu YZ, Sun LP, Liu JW, Gong YH, Xu Q, et al. Epistatic SNP interaction of ERCC6 with ERCC8 and their joint protein expression contribute to gastric cancer/atrophic gastritis risk. Oncotarget.

2017;8(26):43140-52.

15. Stolte M, Meining A. The updated Sydney system: classification and grading of gastritis as the basis of diagnosis and treatment. Canadian journal of gastroenterology = Journal canadien de gastroenterologie. 2001;15(9):591-8.

16. Hamilton S, Aaltonen L. World Health Organization classification of tumours. Pathology and genetics of turnouts of the digestive system. Lyon: IARC Press; 2000. 237-40 p.

17. Santiago JM, Sasako M, Osorio J. [TNM-7th edition 2009 (UICC/AJCC) and Japanese Classification 2010 in Gastric Cancer. Towards simplicity and standardisation in the management of gastric cancer]. Cirugia espanola. 2011;89(5):275-81.

18. Detre S, Saclani Jotti G, Dowsett M. A "quickscore" method for immunohistochemical semiquantitation: validation for oestrogen receptor in breast carcinomas. Journal of clinical pathology. 1995;48(9):876-8.

19. Weinstein JN, Collisson EA, Mills GB, Shaw KR, Ozenberger BA, Ellrott K, et al. The Cancer Genome Atlas Pan-Cancer analysis project. Nature genetics. 2013;45(10):1113-20.

20. Akbani R, Ng PK, Werner HM, Shahmoradgoli M, Zhang F, Ju Z, et al. A pan-cancer proteomic perspective on The Cancer Genome Atlas. Nature communications. 2014;5:3887.

21. Szklarczyk D, Gable AL, Lyon D, Junge A, Wyder S, Huerta-Cepas J, et al. STRING v11: protein-protein association networks with increased coverage, supporting functional discovery in genome-wide experimental datasets. Nucleic Acids Res. 2019;47(D1):D607-d13.

22. Su G, Morris JH, Demchak B, Bader GD. Biological network exploration with Cytoscape 3. Current protocols in bioinformatics. 2014;47:8.13.1-24.

23. Huang da W, Sherman BT, Lempicki RA. Systematic and integrative analysis of large gene lists using DAVID bioinformatics resources. Nature protocols. 2009;4(1):44-57.

24. Subramanian A, Tamayo P, Mootha VK, Mukherjee S, Ebert BL, Gillette MA, et al. Gene set enrichment analysis: a knowledge-based approach for interpreting genome-wide expression profiles. Proc Natl Acad Sci U S A. 2005;102(43):15545-50.

25. Subramanian A, Tamayo P, Mootha VK, Mukherjee S, Ebert BL, Gillette MA, et al. Gene set enrichment analysis: A knowledge-based approach for interpreting genome-wide expression profiles. Proceedings of the National Academy of Sciences. 2005;102(43):15545-50.

26. Warde-Farley D, Donaldson SL, Comes O, Zuberi K, Badrawi R, Chao P, et al. The GeneMANIA prediction server: biological network integration for gene prioritization and predicting gene function. Nucleic Acids Res. 2010;38(Web Server issue):W214-20.

27. Lee JH, Demarest TG, Babbar M, Kim EW, Okur MN, De S, et al. Cockayne syndrome group B deficiency reduces H3K9me3 chromatin remodeler SETDB1 and exacerbates cellular aging. Nucleic Acids Res. 2019;47(16):8548-62.

28. Venema J, Mullenders LH, Natarajan AT, van Zeeland AA, Mayne LV. The genetic defect in Cockayne syndrome is associated with a defect in repair of UV-induced DNA damage in transcriptionally active DNA. Proceedings of the National Academy of Sciences of the United States of America. 1990;87(12):4707-11.

29. Tuteja N, Tuteja R. Unraveling DNA repair in human: molecular mechanisms and consequences of repair defect. Critical reviews in biochemistry and molecular biology. 2001;36(3):261-90.

30. Ganzinelli M, Mariani P, Cattaneo D, Fossati R, Fruscio R, Corso S, et al. Expression of DNA repair genes in ovarian cancer samples: biological and clinical considerations. European journal of cancer (Oxford, England : 1990). 2011;47(7):1086-94.

31. Zhao Z, Zhang G, Li W. Elevated Expression of ERCC6 Confers Resistance to 5-Fluorouracil and Is Associated with Poor Patient Survival in Colorectal Cancer. DNA Cell Biol. 2017;36(9):781-6.

32. Deng N, Liu JW, Sun LP, Xu Q, Duan ZP, Dong NN, et al. Expression of XPG protein in the development, progression and prognosis of gastric cancer. PloS one. 2014;9(9):e108704.

33. Deng Q, Yang H, Lin Y, Qiu Y, Gu X, He P, et al. Prognostic value of ERCC1 mRNA expression in non-small cell lung cancer, breast cancer, and gastric cancer in patients from Southern China. International journal of clinical and experimental pathology. 2014;7(12):8312-21.

34. Fousteri M, Mullenders LH. Transcription-coupled nucleotide excision repair in mammalian cells: molecular mechanisms and biological effects. Cell Res. 2008;18(1):73-84.

Page $10 / 20$ 
35. Boetefuer EL, Lake RJ, Fan HY. Mechanistic insights into the regulation of transcription and transcription-coupled DNA repair by Cockayne syndrome protein B. Nucleic Acids Res. 2018;46(15):7471-9.

36. Pines A, Dijk M, Makowski M, Meulenbroek EM, Vrouwe MG, van der Weegen Y, et al. TRiC controls transcription resumption after UV damage by regulating Cockayne syndrome protein A. Nat Commun. 2018;9(1):1040.

37. Saijo M. The role of Cockayne syndrome group A (CSA) protein in transcription-coupled nucleotide excision repair. Mech Ageing Dev. 2013;134(5-6):196-201.

38. Al-Batran SE, Ducreux M, Ohtsu A. mTOR as a therapeutic target in patients with gastric cancer. Int J Cancer. 2012;130(3):491-6.

39. Riquelme I, Tapia O, Espinoza JA, Leal P, Buchegger K, Sandoval A, et al. The Gene Expression Status of the PI3K/AKT/mTOR Pathway in Gastric Cancer Tissues and Cell Lines. Pathol Oncol Res. 2016;22(4):797-805.

40. Fan QW, Weiss WA. Targeting the RTK-PI3K-mTOR axis in malignant glioma: overcoming resistance. Curr Top Microbiol Immunol. 2010;347:279-96.

41. Bodur C, Kazyken D, Huang K, Ekim Ustunel B, Siroky KA, Tooley AS, et al. The IKK-related kinase TBK1 activates mTORC1 directly in response to growth factors and innate immune agonists. Embo j. 2018;37(1):19-38.

42. Cooper JM, Ou YH, McMillan EA, Vaden RM, Zaman A, Bodemann BO, et al. TBK1 Provides ContextSelective Support of the Activated AKT/mTOR Pathway in Lung Cancer. Cancer Res. 2017;77(18):507794.

43. Hasan M, Gonugunta VK, Dobbs N, Ali A, Palchik G, Calvaruso MA, et al. Chronic innate immune activation of TBK1 suppresses mTORC1 activity and dysregulates cellular metabolism. Proc Natl Acad Sci U S A. 2017;114(4):746-51.

44. Wu G, Feng X, Stein L. A human functional protein interaction network and its application to cancer data analysis. Genome Biol. 2010;11(5):R53.

\section{Tables}

Due to technical limitations, table 2 and table 3 are only available as a download in the Supplemental Files section.

\section{Figures}



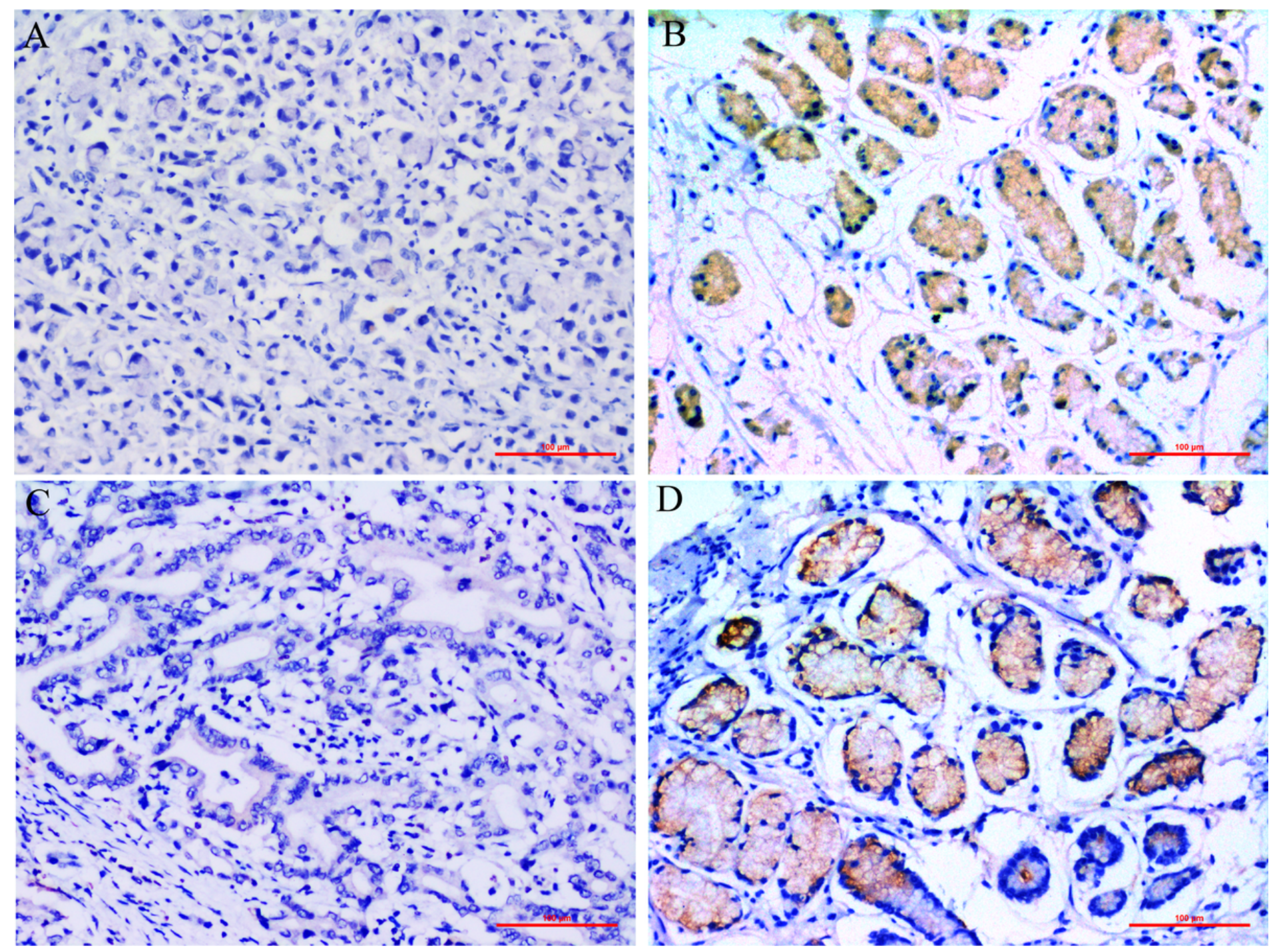

Figure 1

Immunohistochemical staining for ERCC6 and ERCC8 expression in GC and its paired pan-cancerous normal tissues. A. GC with negative ERCC6 expression; B. paired pan-cancerous normal tissues with positive ERCC6 expression; C. GC with negative ERCC8 expression; D. paired pan-cancerous normal tissues with positive ERCC8 expression. (Magnification $\times 200$; bar $=100 \mu \mathrm{m}$ ) 

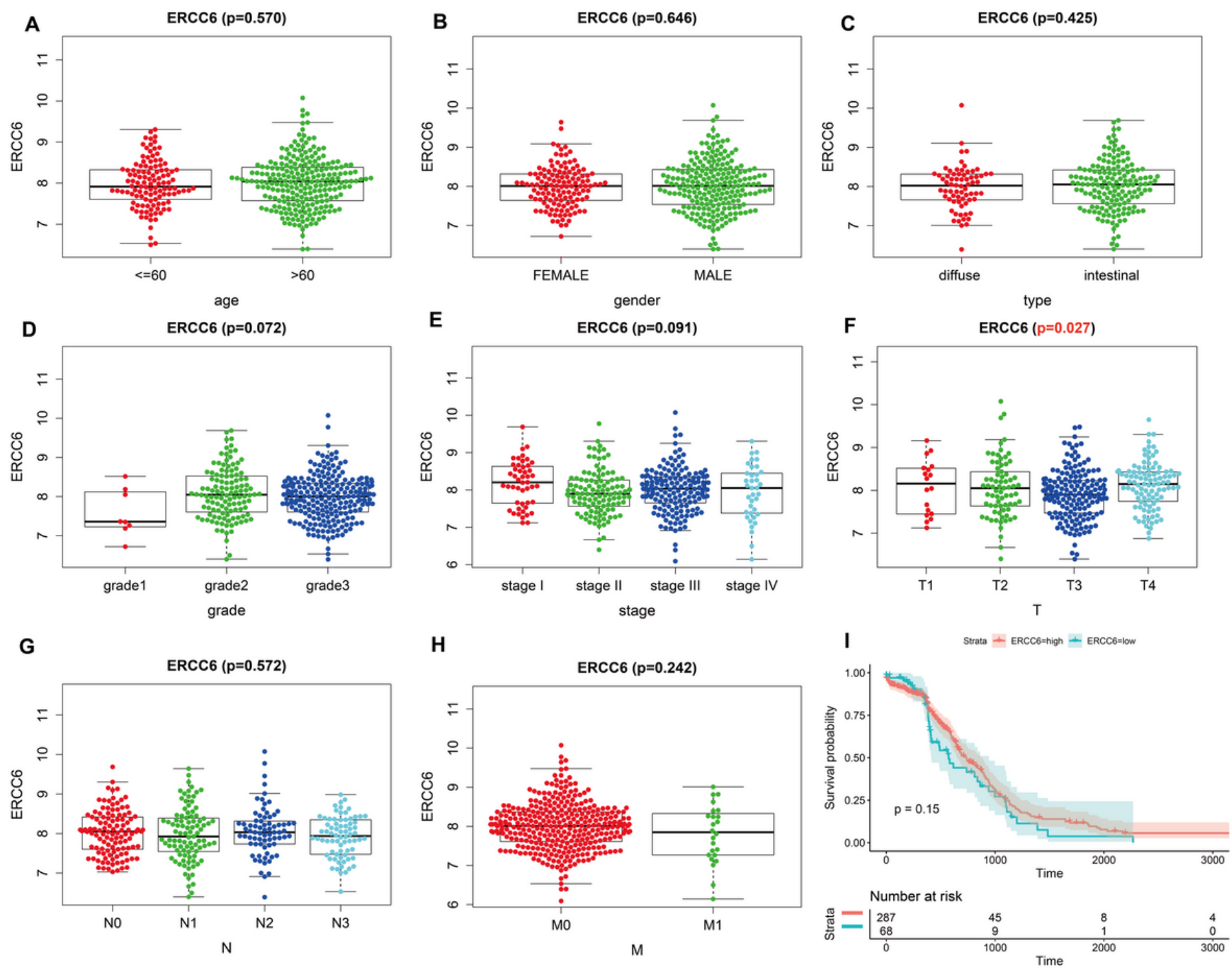

Figure 2

Correlation analysis of ERCC6 expression with clinicopathological parameters and survival of GC using TCGA data. (A)age, (B)gender, (C)type, (D)grade, (E)stage, $(F)$ T stage, $(\mathrm{G}) \mathrm{N}$ stage, $(\mathrm{H}) \mathrm{M}$ stage, (I)univariate survival analysis. A $P<0.05$ was deemed statistically significant. 

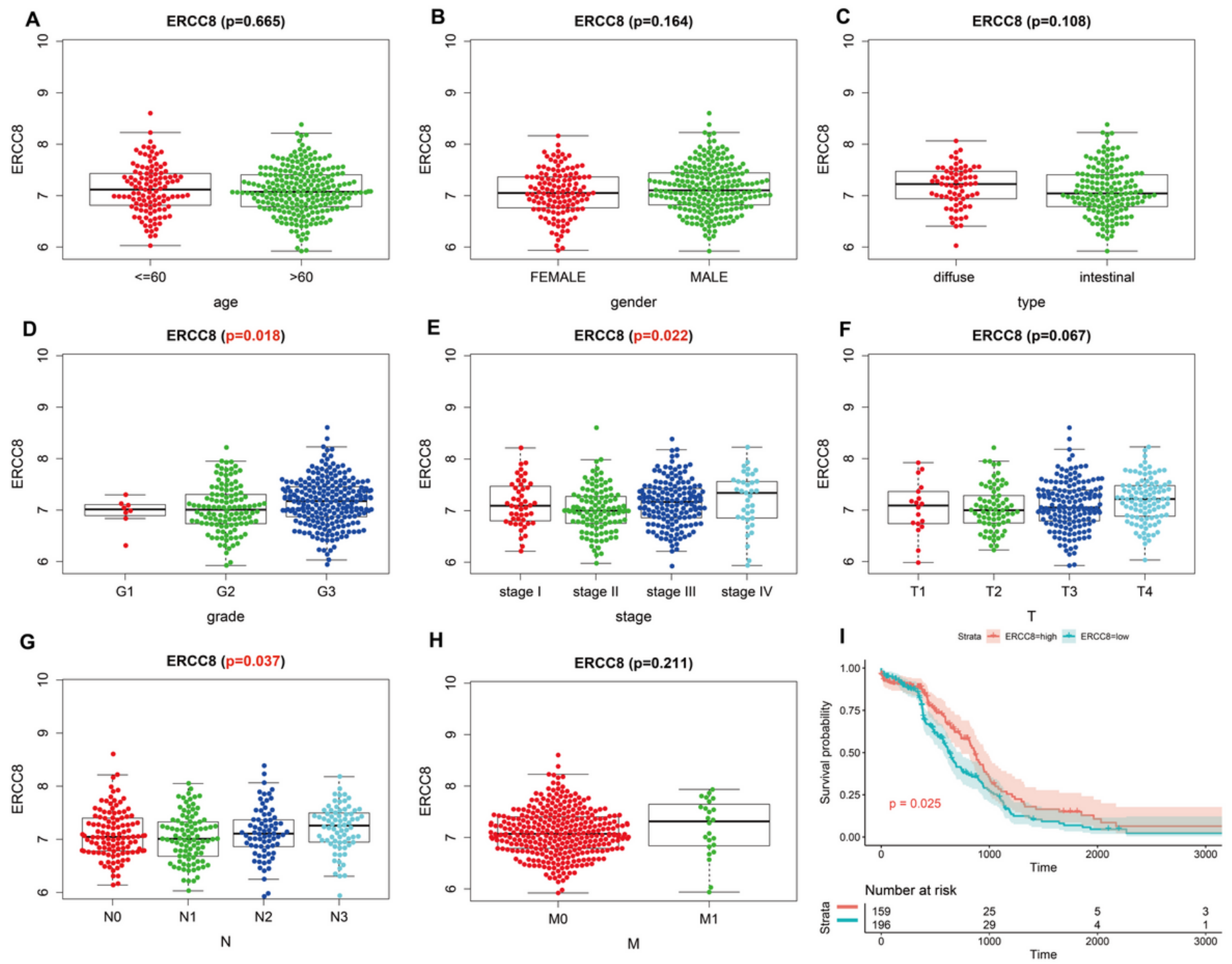

Figure 3

Correlation analysis of ERCC8 expression with clinicopathological parameters and survival of GC using TCGA data. (A)age, (B)gender, (C)type, (D)grade, (E)stage, (F)T stage, (G)N stage, (H)M stage, (I) univariate survival analysis. A P $<0.05$ was deemed statistically significant. 


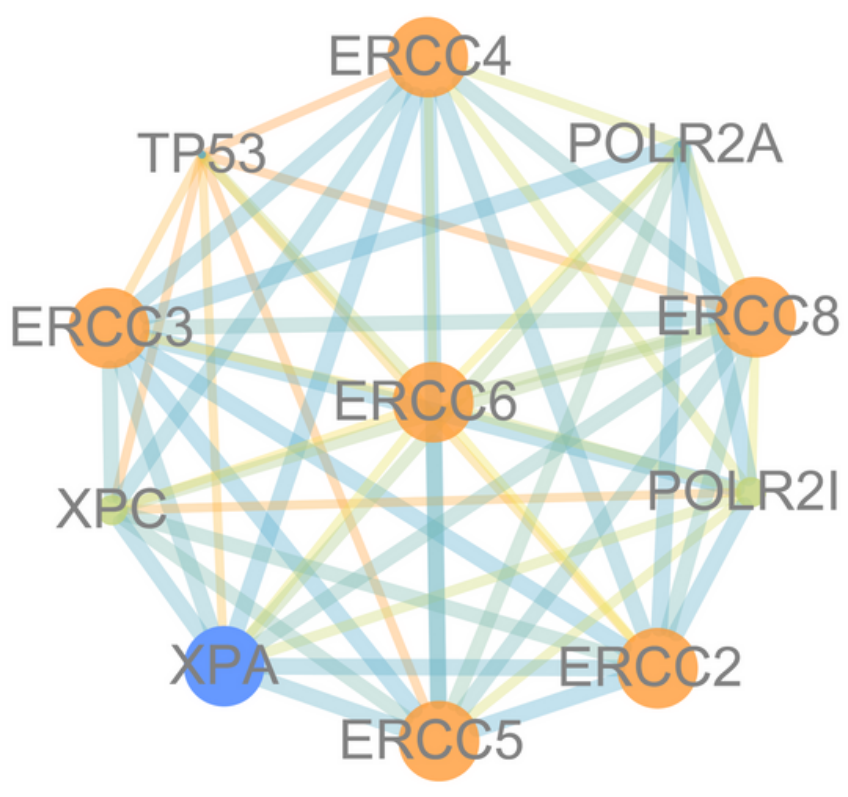

A

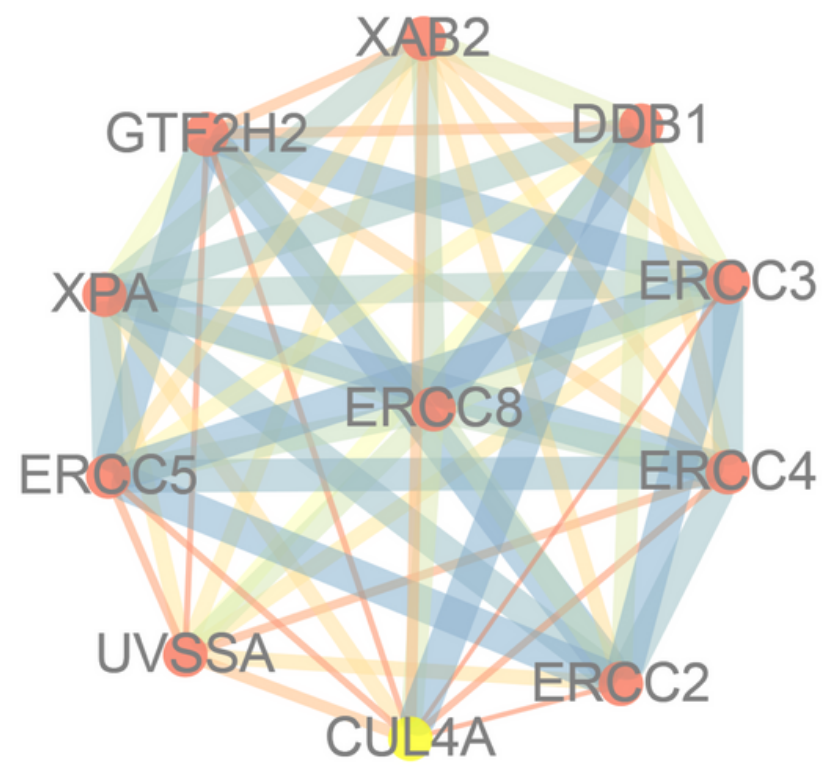

B

\section{Figure 4}

Protein interaction networks of 10 associated partners with a confidence score $>0.4$ obtained from String database. A. ERCC6 is defined as the core gene; B. ERCC8 is defined as the core gene. 


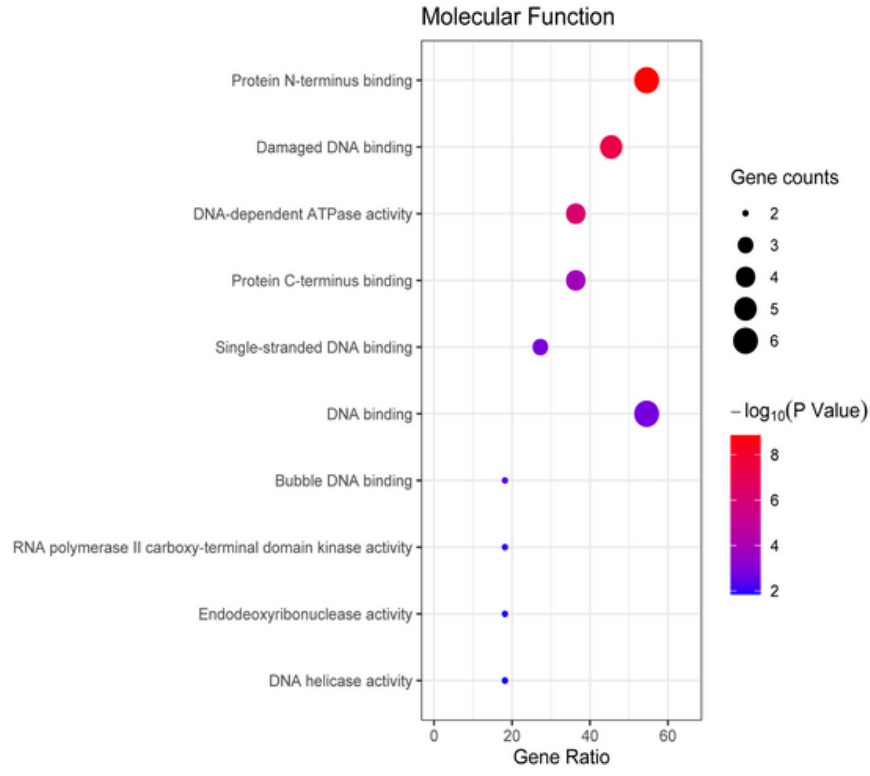

A

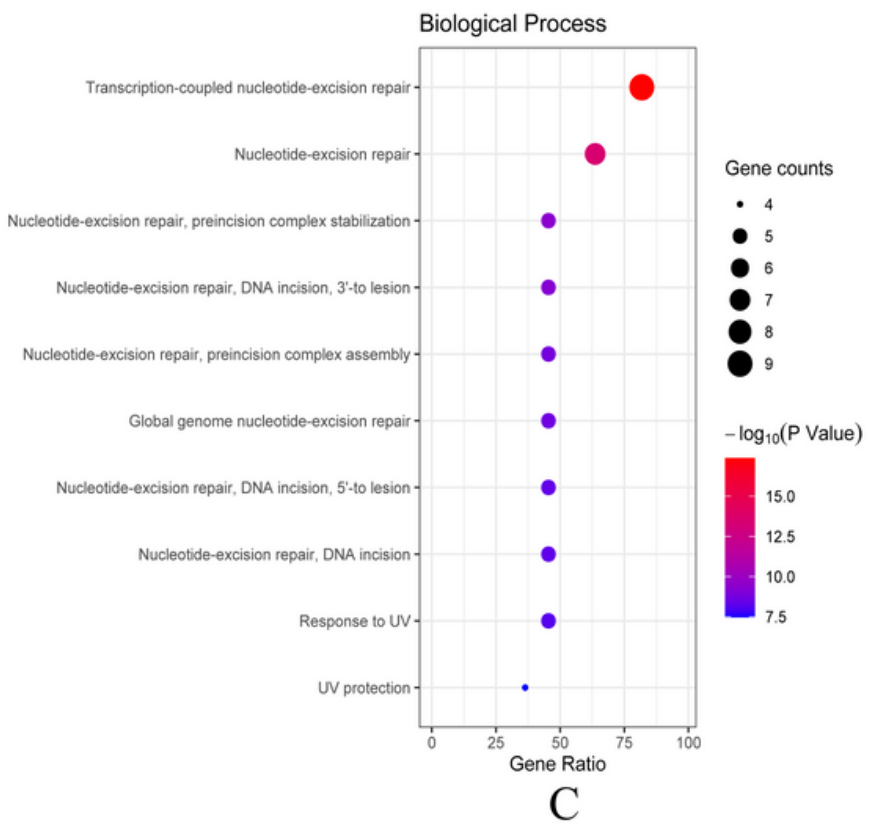

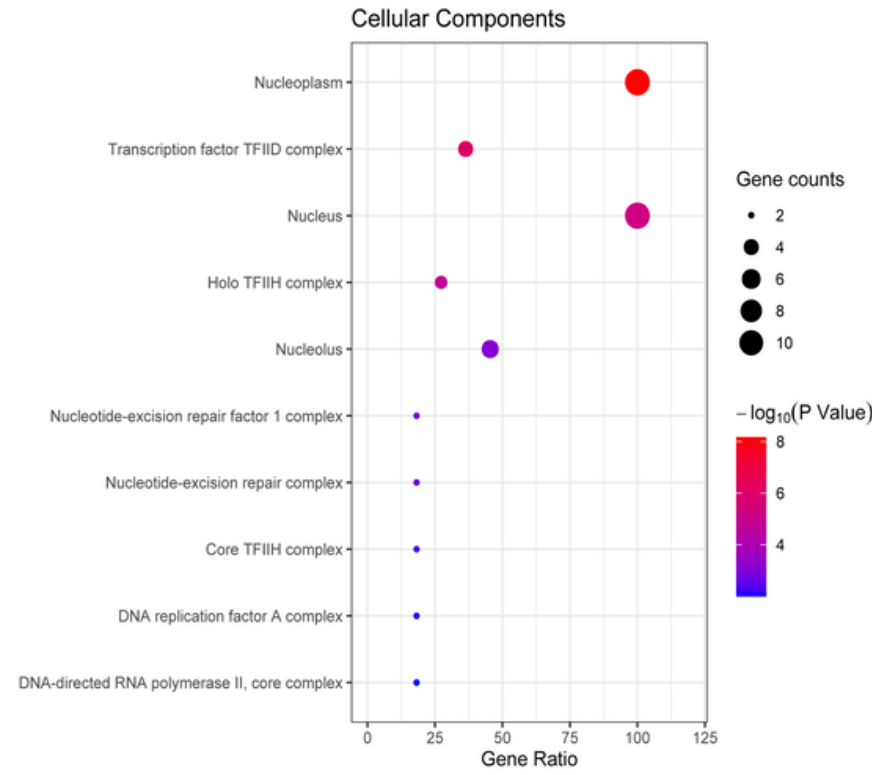

B

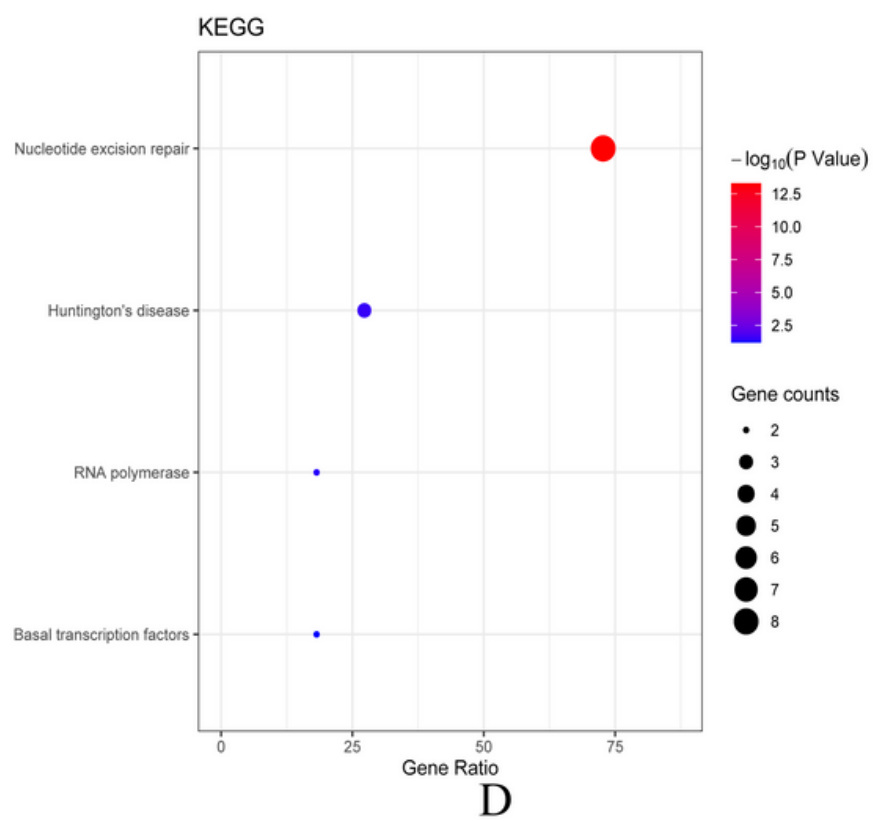

\section{Figure 5}

The bubble diagram of enrichment and pathway analysis of ERCC6 network genes. A. Top ten categories for molecular function of $\mathrm{GO}$ analysis; B. Top ten categories cellular component of GO analysis; $\mathrm{C}$. Top ten categories for biological process of GO analysis; D. KEGG pathway analysis results. 


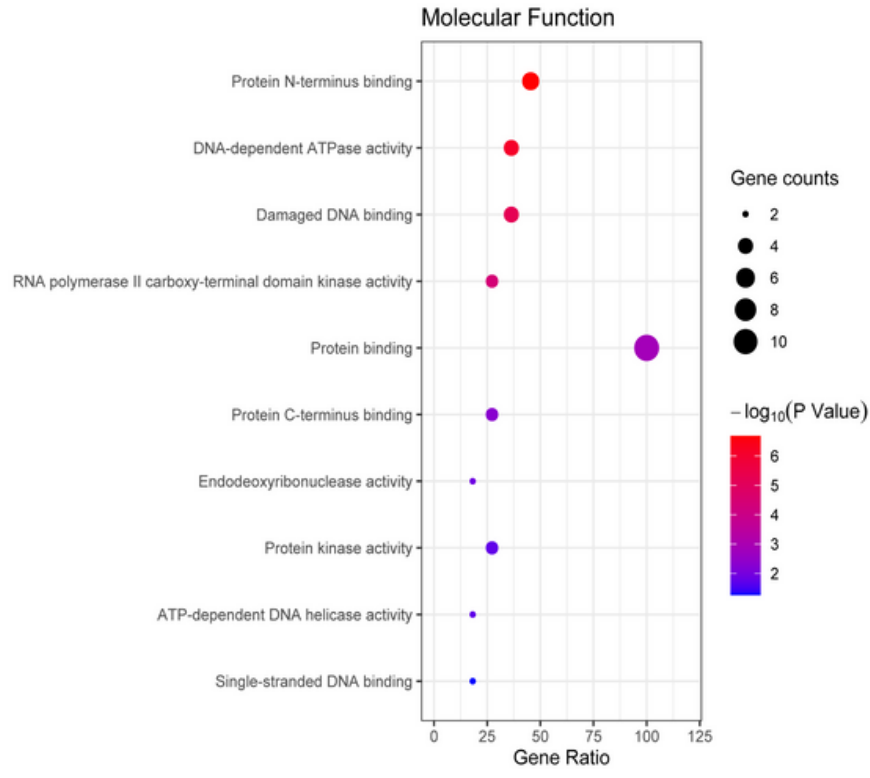

A

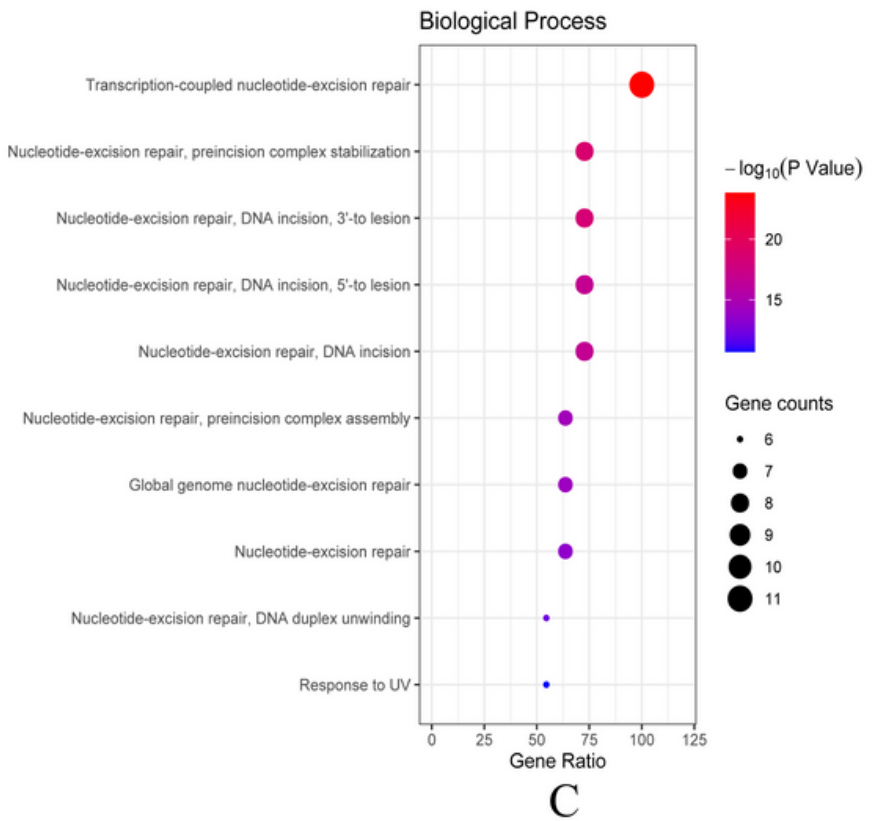

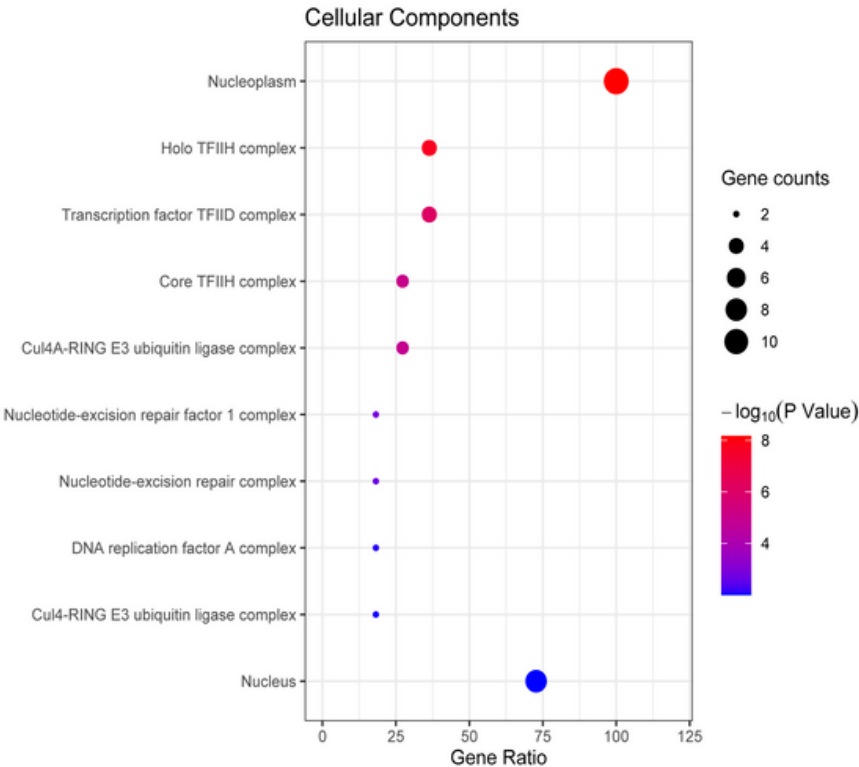

B

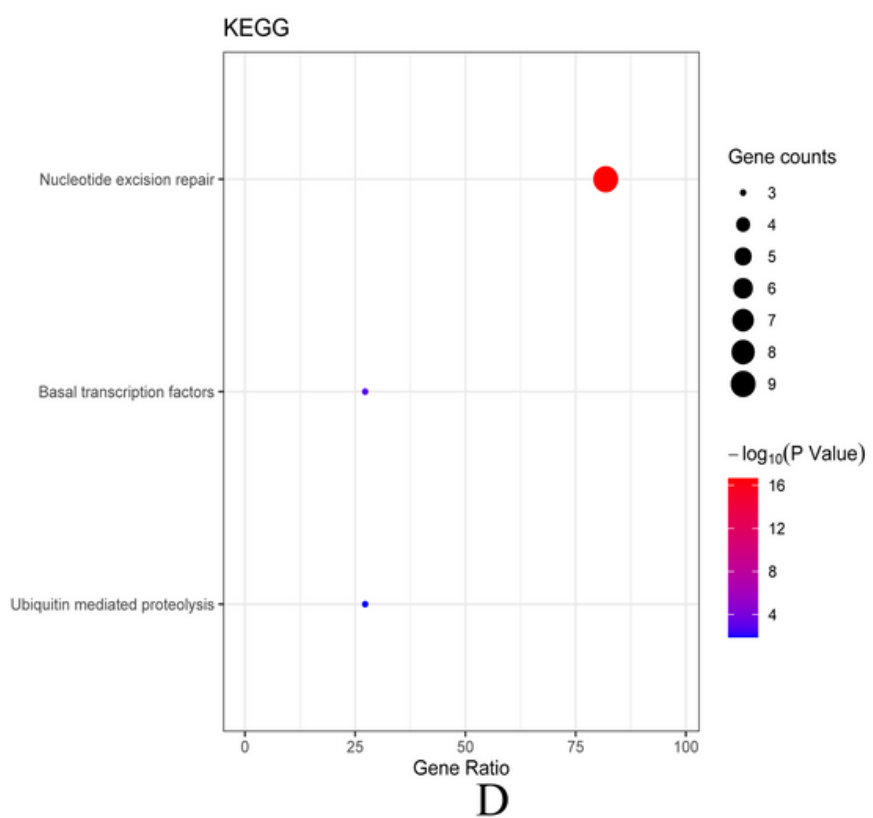

\section{Figure 6}

The bubble diagram of enrichment and pathway analysis of ERCC8 network genes. A. Top ten categories for molecular function of GO analysis; B. Top ten categories cellular components of GO analysis; C. Top ten categories for biological process of GO analysis; D. KEGG pathway analysis results. 

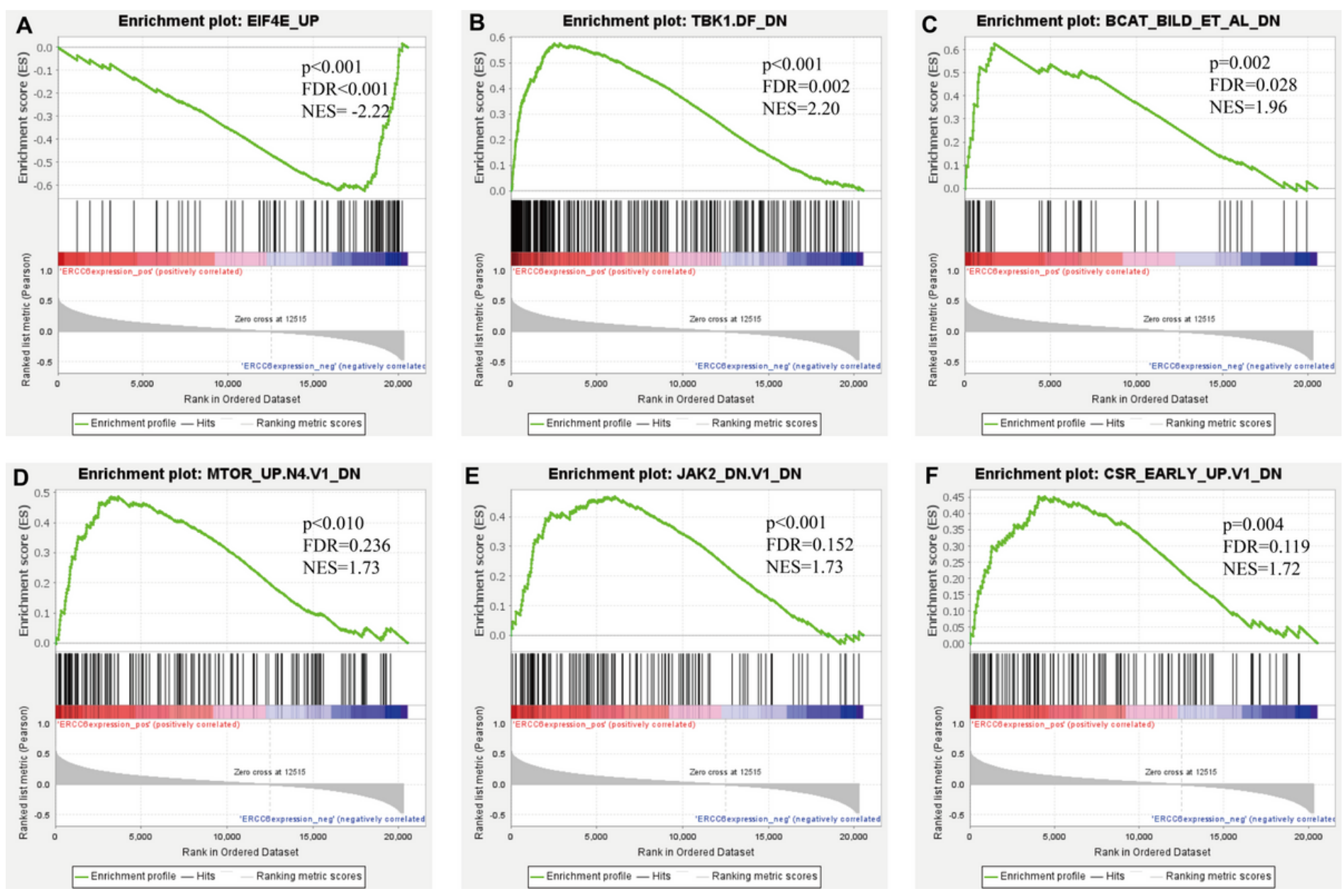

\section{Figure 7}

GSEA analyses results for ERCC6 in GC patients. GSEA results showing nine high-scoring gene sets including (A)EIF4E UP, (B)TBK1.DF DN, (C) BCAT BILD ET AL DN, (D) MTOR UP.N4.V1 DN, (E) JAK2 DN.V1 DN, (F) CSR EARLY UP.V1 DN are differentially enriched in ERCC6-related GC. NES, normalized enrichment score; FDR, false discovery rate.
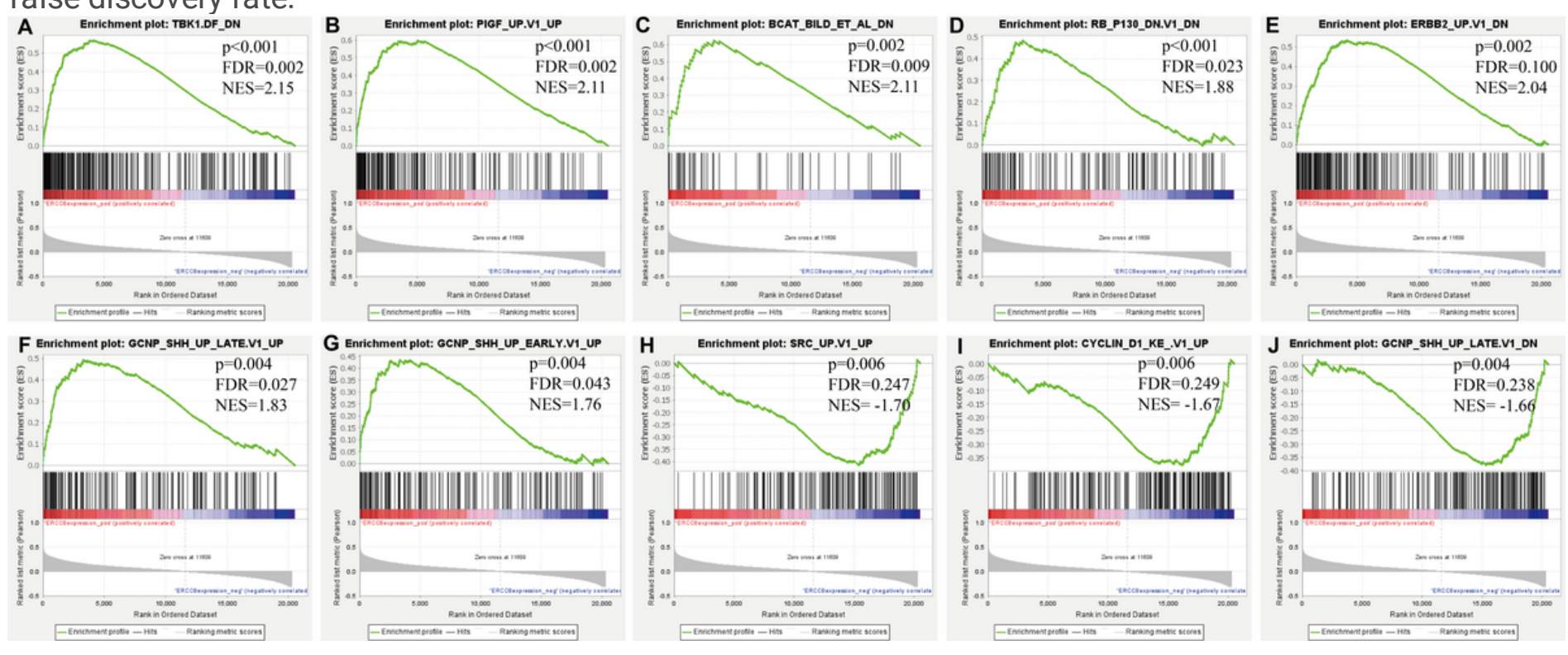

Figure 8 
(A)TBK1.DF DN, (B)PIGF UP.V1 UP, (C)BCAT BILD ET AL DN, (D)RB P130 DN.V1 DN, (E)ERBB2 UP.V1 DN,

(F) GCNP SHH UP LATE.V1 UP, (G)GCNP SHH UP EARLY.V1 UP, (H)SRC UP.V1 UP, (I)CYCLIN D1 KE.V1 UP and (J) GCNP SHH UP LATE.V1 DN are differentially enriched in ERCC8-related GC. NES, normalized enrichment score; FDR, false discovery rate.
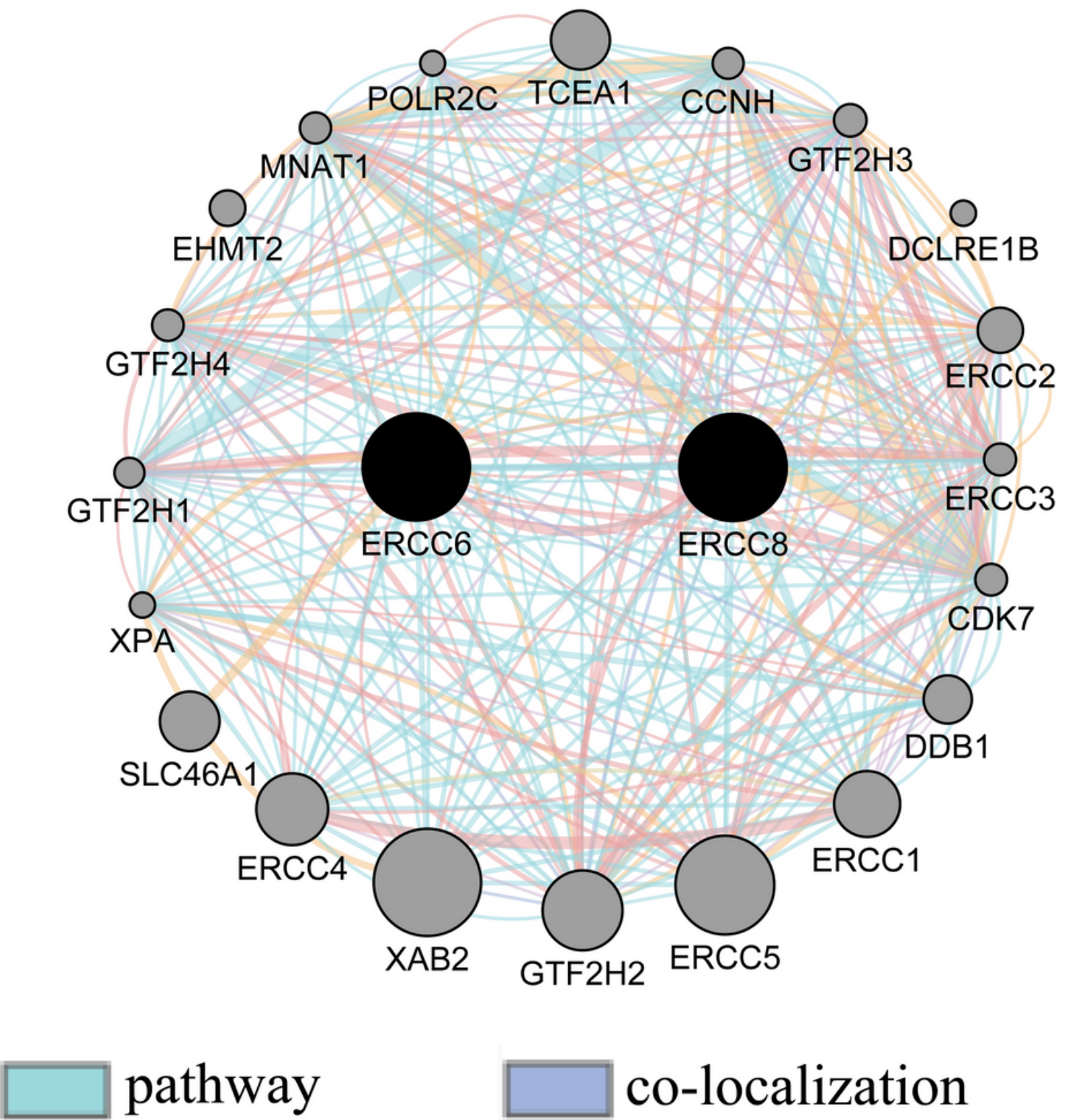

pathway

$\square$ co-localization

predicted

physical interactions

co-expression

shared protein domains

Figure 9

Gene-gene interaction network between ERCC6 and ERCC8. Nodes and links represent genes and networks respectively.

\section{Supplementary Files}


This is a list of supplementary files associated with this preprint. Click to download.

- Supplementaryfile1.xlsx

- Supplementaryfile2.xlsx

- Table1.xlsx

- table2.xlsx 